\title{
Large-Eddy Simulation of a Wing-Body Junction Flow
}

\author{
Sungmin Ryu,,- Michael Emory, \pm Gianluca Iaccarino,, \pm and Alejandro Campos $\stackrel{\S}{\$}$ \\ Stanford University, Stanford, California 94305 \\ and \\ Karthik DuraisamyI \\ University of Michigan, Ann Arbor, Michigan 48109 \\ DOI: $10.2514 / 1 . J 054212$
}

\begin{abstract}
Large-eddy simulations of a wing-body junction flow experimentally studied are presented. Wall junction flows are common in engineering applications, but the flow physics at the corners of the junction is not well understood. Moreover, in these types of flows, the performance of the subgrid-scale models frequently used for large-eddy simulations is not well characterized. To address these issues, large-eddy simulations of the wing-body junction are performed, with multiple levels of grid resolution. Two-dimensional turbulent profiles are generated every time step and introduced to the inlet plane of the computation domain to mimic the unsteady turbulent boundary layer. A Reynolds-averaged Navier-Stokes calculation is used as a precursor to initialize the flowfield of the large-eddy simulations. Then, large-eddy simulations with the Vreman model is performed and the simulation results are analyzed with respect to three specific goals: compare the computed and measured turbulence statistics in the junction region, investigate the flow physics in the corner region, and discuss the potential reasons for the inaccuracies of the subgrid-scale model. The sensitivity of the predicted results to inflow conditions and to subgrid-scale modeling is also investigated.
\end{abstract}

\section{Nomenclature}

$C_{p} \quad=$ mean pressure coefficient

$c \quad=$ chord length of airfoil, $\mathrm{m}$

$k=$ turbulent kinetic energy, $\mathrm{m}^{2} / \mathrm{s}^{2}$

Re $\quad=$ Reynolds number

$T=$ maximum thickness of airfoil, $\mathrm{m}$

$T_{w} \quad=\quad$ wall temperature, $\mathrm{K}$

$U, V, W=$ mean velocity in $x, y$, and $z$ directions, $\mathrm{m} / \mathrm{s}$

$u, v, w=$ transient velocities in $x, y$, and $z$ directions, $\mathrm{m} / \mathrm{s}$

$x, y, z=$ Cartesian axes, $\mathrm{m}$

$\Delta \quad=$ grid spacings

$\theta \quad=$ momentum thickness, $\mathrm{m}$

$\rho \quad=$ density, $\mathrm{kg} / \mathrm{m}^{3}$

$\Omega \quad=$ vorticity, $1 / \mathrm{s}$

$\omega=$ specific turbulence dissipation rate, $1 / \mathrm{s}$

\section{Subscripts}

$\begin{array}{lll}n & = & \text { surface normal direction } \\ s & = & \text { spanwise direction } \\ t & = & \text { surface tangential direction }\end{array}$

\section{Superscripts}

$$
\begin{array}{lll}
1 & = & \text { disturbance quantity } \\
+ & = & \text { quantity in wall unit }
\end{array}
$$

Received 28 January 2015; revision received 11 June 2015; accepted for publication 7 October 2015; published online 20 January 2016. Copyright $(\subset)$ 2015 by the American Institute of Aeronautics and Astronautics, Inc. All rights reserved. Copies of this paper may be made for personal or internal use, on condition that the copier pay the $\$ 10.00$ per-copy fee to the Copyright Clearance Center, Inc., 222 Rosewood Drive, Danvers, MA 01923; include the code 1533-385X/15 and $\$ 10.00$ in correspondence with the CCC.

*Postdoctoral Scholar, Department of Mechanical Engineering.

${ }^{\dagger}$ Postdoctoral Scholar, Department of Mechanical Engineering, Center for Turbulence Research. Member AIAA.

ॠAssociate Professor, Department of Mechanical Engineering. Member AIAA.

${ }^{\S}$ Ph.D. Candidate, Department of Aeronautics and Astronautics. Student Member AIAA.

${ }^{\top}$ Assistant Professor, Department of Aerospace Engineering. Member AIAA.

\section{Introduction}

B ODY junction flows around wall-mounted obstacles frequently occur in various engineering situations, for example, an aircraft wing-body junction or a turbine blade-hub flow. These flows are subject to a strong adverse pressure gradient in the streamwise and/or lateral directions, depending on the shape of the obstacle. As a consequence, three-dimensional (3-D) turbulent boundary layers, complex recirculation zones, and strong coherent vortical structures, such as horse shoe vortices (HSVs) are present. The complex structure of junction flows can result in undesirable effects (e.g., increasing drag, heat transfer, and surface pressure fluctuation near the junction), as discussed by Simpson [1]. It is important to predict such flows correctly to both better understand the physics of junction flows and use simulations for improving their design. Moreover, 3-D pressure-driven turbulent boundary layers, such as that in a wingbody junction, can serve as effective benchmarks to assess and develop turbulence models.

Devenport and Simpson [2] carried out experimental measurements in the separated-flow region near the nose of a cylindrical wing, made up of a 3:2 semi-elliptic nose and NACA 0020 tail, protruding from a flat surface using three-component laser anemometry. Specifically, they presented low-frequency oscillations of the HSV, which they called bimodal dynamics. Fleming et al. [3] also measured velocities adjacent to the wing, as well as wake regions far downstream, investigating spanwise flow patterns induced by a lateral adverse pressure gradient. To study the anisotropy of the nearwall turbulence quantities, Ölçmen and Simpson [4] experimentally measured velocities along stations at which strong lateral cross flows were present.

Numerical studies have been extensively performed on selected experimental configurations. In the case of Reynolds-averaged Navier-Stokes (RANS) calculations, turbulence models have been actively assessed in their capability to represent the strongly anisotropic behaviors of the separated boundary layers near the junction. Apsley and Leschziner [5] compared the predictive performance of 12 turbulence models, classified into three groups (linear, nonlinear, and differential stress models) on wing-body junction flows. Although second moment closure shows better agreement with experiments than other models in their study, none of the models could correctly capture mean flow structures and turbulence statistics near the nose of the wing. Parneix et al. [6] performed RANS calculation of wing-body junction flows to validate the $\mathrm{V} 2 \mathrm{~F}$ model. Based on a detailed comparison of the turbulent statistics with those of Devenport and Simpson [2] and 
Ölçmen and Simpson [4], they demonstrated that the V2F model with the linear eddy-viscosity hypothesis adequately captures the 3-D separation line, the location of HSVs, and its intensity [6]. The original V2F model, used in Lien et al. [7], was found to introduce numerical convergence issues due to the boundary conditions used for the " $f$ " variable. A modified version of the V2F model was developed to remove this problem in Lien et al. [7] and Iaccarino [8]. Although successful in reproducing two-dimensional (2-D) separated flows, the modifications did not produce accurate results for the wing-body junction flow. However, as discussed by Devenport and Simpson [2], and Simpson [1], RANS calculations are inherently limited in their ability to capture either the turbulent anisotropy generated near the junction or the transient behavior of the separated flow and corresponding vortical structure [specifically, the bimodal probability density function (PDF) of the velocity fluctuations].

To resolve unsteady small- and large-scale motions of junction flows, Paik et al. [9] performed detached-eddy simulations (DESs) targeting the experimental measurements of Devenport and Simpson [2]. They adjusted the turbulence length scale to overcome the shortcomings of the original DES, namely, the presence of a laminarlike separation [9]. As a consequence of the adjusted length scale, the modified DES captures the shape of the HSV, its core location, and the bimodal distribution of the PDF of streamwise velocity fluctuations better than the original DES. Gand et al. [10] investigated junction flows between a NACA 0012 airfoil and a flat plate, with a Reynolds number based on chord length and freestream velocity of $R e_{c}=2.8 \times 10^{5}$. They presented the unsteadiness of the HSV, noting that frequencies associated with the motion of the legs of the HSV are higher than those of the HSV near the wing nose in the plane of symmetry. However, they also noted that the $R e_{\theta}$ at the incoming boundary layer in their wing-body junction case was not sufficiently high to represent realistic wing-body junction conditions. As a reference, the $R e_{\theta}$ in the experiments performed by Devenport and Simpson [2] is about 5960.

Researches about corner flows have been performed, experimentally and numerically. Pierce et al. [11,12] suggested experimental evidence of the existence of a tiny counter-rotating corner vortex (paired with a dominant junction vortex) by computing a transverse vorticity field in the symmetry plane (see figure 9 in $[11,12])$. Simpson [1] mentioned various systems of vortical structures in the symmetry plane of laminar and turbulent junction flows and a small corner vortex is depicted in the systems (see figure 2 in [1]). Recently, Gand et al. [10] investigated corner flow separations near the trailing edge around a wall-mounted airfoil, numerically and experimentally. However, mean flow structure and Reynolds stress anisotropy at the corner region in the symmetry plane were not discussed in the papers.

In this paper, we describe the large-eddy simulation (LES) investigation of the wing-body junction flow experimentally studied by Devenport and Simpson [2]. The LES calculation is validated by comparisons of the mean flow structure and turbulence statistics with experimental data in Secs. III.A-III.F. Moreover, corner flows are analyzed in their transient behavior, turbulence anisotropy, and generation mechanism. In Sec. IV, the sensitivity of the predicted turbulence statistics to the generated inlet conditions and SGS modeling is scrutinized in LES performed with a coarse grid.

\section{Numerical Setup}

The flow around the wall-mounted wing on a solid wall is numerically investigated at $R e_{T}=1.15 \times 10^{5}$ based on the maximum thickness and the approaching bulk velocity. Figure $1 \mathrm{a}$ displays the geometry of the computational domain. Table 1 shows the near-wall grid resolution in the tangential, normal, and spanwise directions. The compressible Navier-Stokes equations for LES are solved by a finite-volume-based in-house code CharlesX [13]. A third-order Runge-Kutta scheme is used for time integration, and spatial derivatives for the flow variables are approximated using the second-order blend of nondissipative central and dissipative upwind fluxes. The Vreman model [14] is used as a baseline model for the

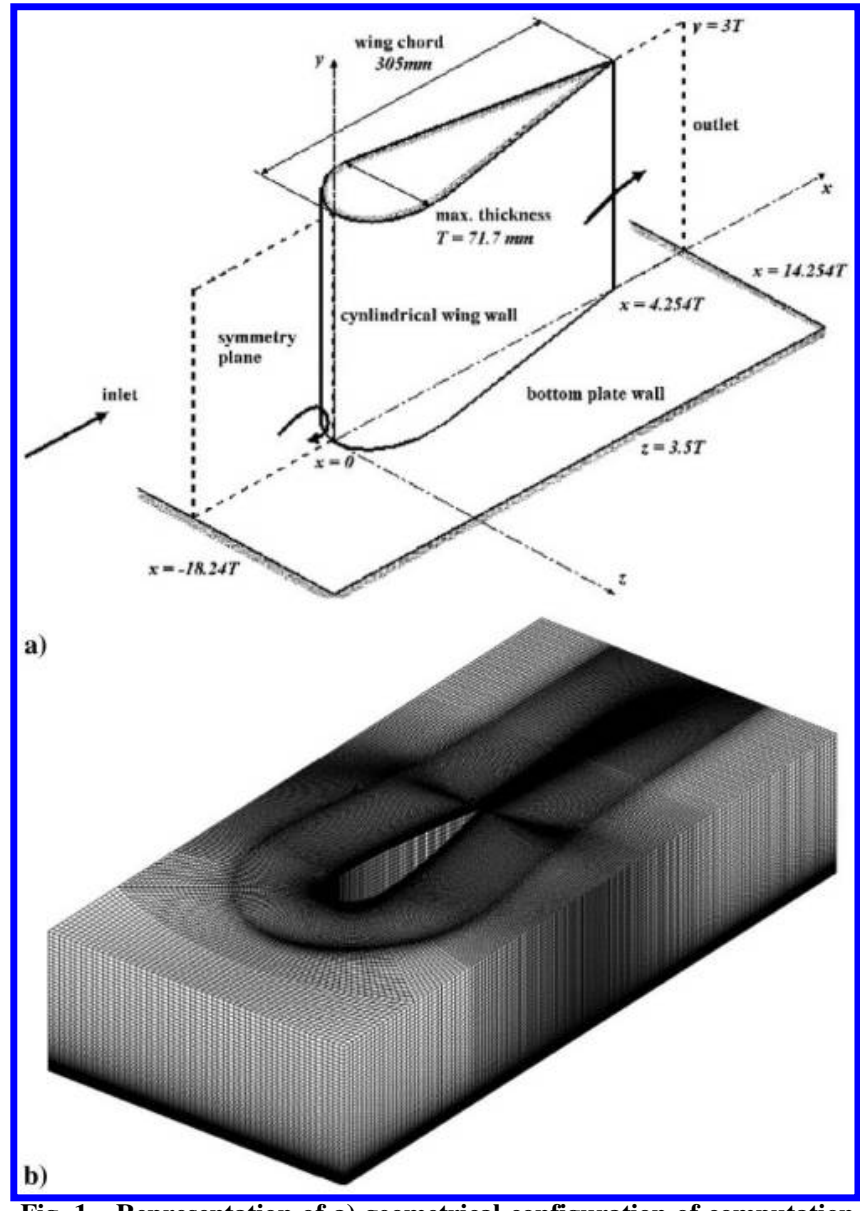

Fig. 1 Representation of a) geometrical configuration of computation domain according to [2], and b) coarse grid with about 5.6 million cells.

unclosed subgrid-scale stress terms. The number of cells for the coarse and fine grids is about $5.6 \times 10^{6}$ and $45 \times 10^{6}$, respectively. The geometry of the computational domain and physical parameters are based on the experimental conditions of Devenport and Simpson [2]. The wall-mounted cylindrical wing consists of a 3:2 elliptical wing nose and a NACA 0020 tail. The maximum thickness of the wing is $T=71.7 \mathrm{~mm}$ and the chord length is $c=4.254 T$.

The locations of boundaries in the computational domain are depicted in Fig. 1a. The inlet and outlet planes are located at $x / T=$ -4.254 and 6.381 . The lateral sides of the computation domain are located at $x / T=-3.5$ and 3.5 . The reduced computational grid (coarse) in the streamwise direction is illustrated in Fig. 1b. At the inlet, 2-D turbulent flows are synthesized using the methodology proposed by Xie and Castro [15], with additional modifications proposed by Touber and Sandham [16]. For the synthesization of 2-D inlet turbulent profiles, one-dimensional, fully developed profiles with $R e_{\theta}=5811$ were calculated using the stress- $\omega$ Wilcox model [17]. The $R e_{\theta}$ at $x / c=-1$ is approximated from the relation between momentum thickness and streamwise development length, $\theta / x=0.036 R e_{x}^{-0.2}$, based on the profile at $R e_{\theta}=5940$ presented by Ölçmen and Simpson [4] at $x / c=-0.75$. Figure 2 shows the inlet plane of the computational domain and the most upstream location of the experimental measurement in Ölçmen and Simpson [4] . The pressure-relaxation characteristic boundary condition is applied to the outlet. A symmetry boundary condition is used at the top and the spanwise boundaries. A no-slip and isothermal boundary condition $\left(T_{w}=298 \mathrm{~K}\right)$ is applied to the bottom wall and the wing surface.

A RANS calculation of the wing-body junction flow is performed on the coarse grid using the $k-\omega$ shear stress transport (SST) model [18] to initialize the flowfield of the LES. The in-house code called Joe [19] is used for the RANS simulation. Boundary conditions for the calculation are those described in Sec. II. Figure $\underline{3}$ shows the iteration history of the logarithmic residuals of the mean flow 
Table 1 Minimum and maximum grid spacings of coarse grid LES in a wall unit in normal, tangential, and spanwise directions, respectively

\begin{tabular}{lccc}
\hline \hline & $\Delta_{n}^{+}$ & $\Delta_{t}^{+}$ & $\Delta_{s}^{+}$ \\
\hline Airfoil & $0.02-1.23$ & $1.83-28.09$ & $0.01-132$ \\
Flat plate & $0.02-2.32$ & $0.13-276$ & $0.1-263$ \\
\hline \hline
\end{tabular}

variables, which are well converged. The RANS results are interpolated onto the coarse and fine grids of the LES to initialize the flowfield. The initialization with RANS results can smoothly induce flow separations near the junction and further a dynamic equilibrium state. The RANS results are also used later as a reference.

\section{Results}

\section{A. Mean Pressure Distribution}

Figure 4 displays comparisons of the mean wall-pressure coefficient in the vicinity of the wing nose between experimental measurements [2] and each simulation result. The RANS and LES show fairly good agreement with the measurements, but predict the locally low pressure (shown on the airfoil surface at $x / T=0.8$ ) further upstream at $x / T=0.6$ compared with the experiment. Moreover, the RANS result shows more clearly kinked contour lines along the wing nose, which is not clearly evident in the LES results or the experiments.

\section{B. Mean Flow Visualization on the Bottom Wall}

Figure 5 shows flow visualizations mimicking the oil flow visualization experimentally performed by Ölçmen and Simpson [4]. The separation line forms ahead of the wing nose and passes along the side of the wall-mounted airfoil. The separation line is generated by the adverse pressure gradient and originates at the separation point $(x / T=-0.47)$. Flow separation near the wake region is also observed with a fishtail-like structure. The RANS is seen to accurately represent the separation line, which branches around

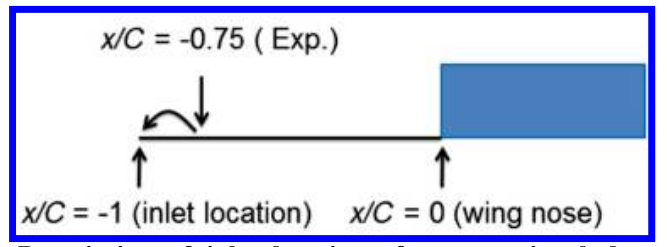

Fig. 2 Description of inlet location of computational domain and most upstream location of experimental measurement in Ölçmen and Simpson [4].

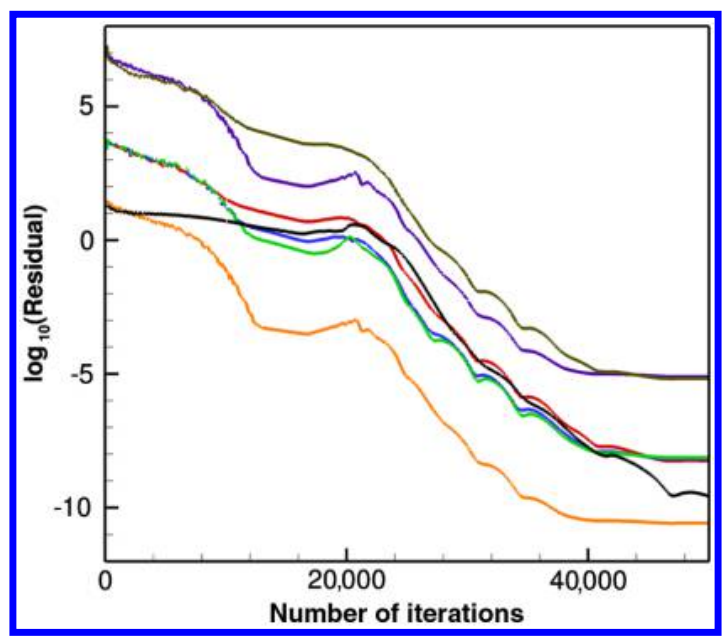

Fig. 3 Semilog plot of residuals of mean flow variables with respect to number of iterations. $x / T=-0.5$. However, the predicted flow separation near the trailing edge appears to be excessive compared with the oil flow visualization, and the fishtail-like shape is not observed. The LES computation predicts fairly well the separation line originating at $x / T=-0.5$ and the flow separation near the trailing edge. The secondary separation line close to the wing nose is also observed in both simulation results. Devenport and Simpson [2] mentioned that there exists a small secondary separation close to the corner region, which was observed in the DES result by Paik et al. [9]. However, no analysis of this corner separation and flow structure is presented in the DES or the experimental results. Corner flows relevant to the secondary separation are analyzed in Sec. III.E using the LES data.

\section{Flow Structures near the Junction}

Figure 6 displays the time-averaged vorticity in the symmetry plane upstream of the airfoil calculated using LES with a fine grid, RANS with the SST $k-\omega$, and DES by Paik et al. [9]. In the experimental measurements by Devenport and Simpson [2], an elliptical mean flow structure is observed and it is slightly lifted up from the bottom wall. This characteristic mean flow behavior is the result of the reverse flows generated by the adverse pressure gradient. Devenport and Simpson [2] described the "bimodal dynamics of the junction vortex," which consist of zero- and backflow modes. The zero-flow mode is generated when the approaching flow encounters the reverse flow near the flat plate and those two flows are ejected toward the freestream region. Consequently, the mean flow structure is slightly lifted, as shown in the experimental data reported in Fig. $\underline{6}$. This is not captured by the RANS calculation with the SST $k-\bar{\omega}$ model, whereas the DES performed by Paik et al. [9] captures this lifted vortex structure. However, the DES predicted the core of the elliptic flow structure further upstream $(x / T=-0.36)$ than the experiment $(x / T=-0.2)$. No quantitative comparisons of mean velocity and turbulence quantities near the separation location between the DES and experimental measurement are presented. The

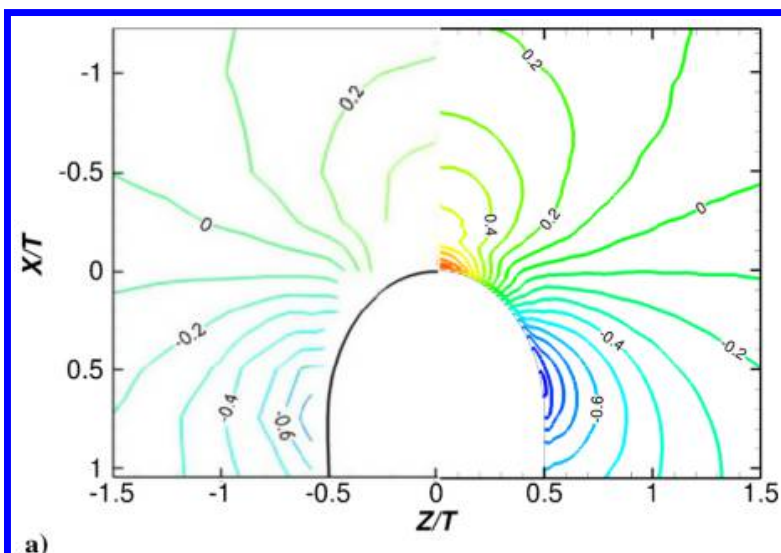

a)

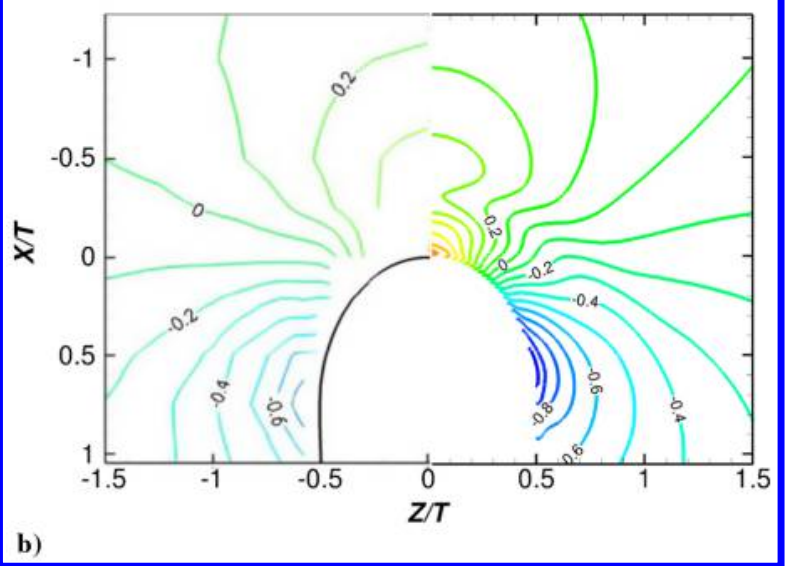

Fig. 4 Mean wall-pressure coefficient $C_{p}$ near junction: a) comparison between experimental measurements (left-half plane) by Devenport and Simpson [2] and LES (right-half plane), and b) experiment vs RANS. 


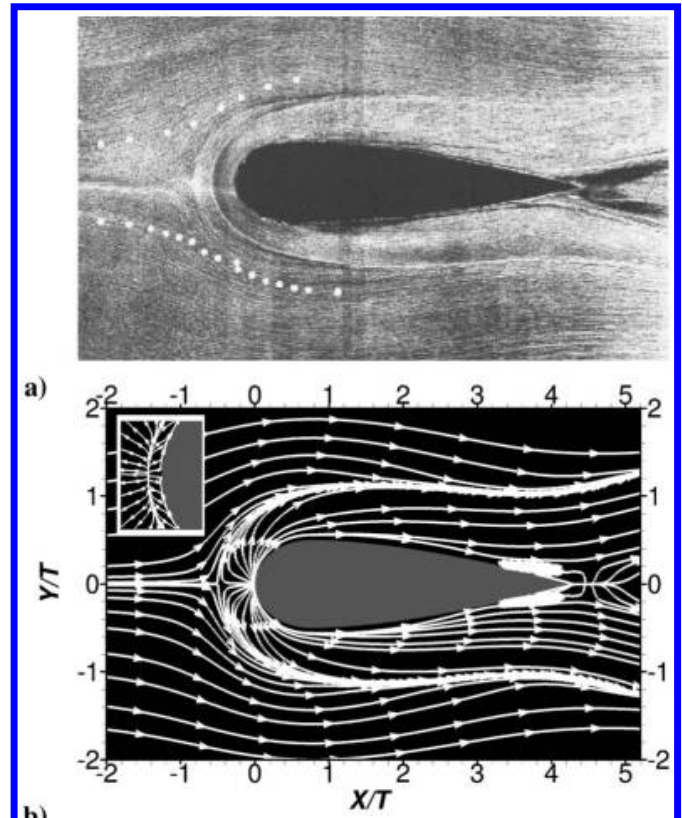

b)

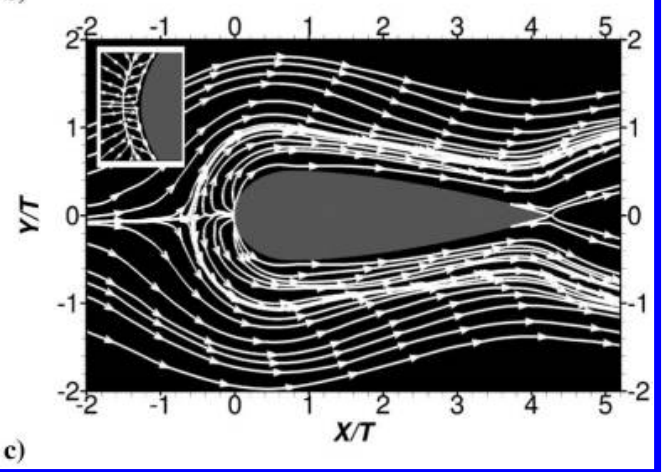

Fig. 5 Representation of a) oil flow visualization on the bottom wall in the experiments by Ölçmen and Simpson [4], and mean flow visualization by friction lines using b) RANS, and c) LĒS.

present LES, performed with a fine grid, captures the characteristic shape of the junction vortex structure and its core location.

\section{Turbulence Statistics}

Turbulence statistics are extracted at the 11 stations located in the symmetry plane upstream of the airfoil, as depicted in Fig. 7. Figure $\underline{8}$ shows quantitative comparisons of the mean streamwise velocity profiles between coarse grid RANS and LES and fine grid LES. The LES computations predict well the separation location at the fifth station, but slightly underpredict the amount of reverse flow induced by the adverse pressure gradient. The RANS calculation predicts a separation location further upstream with a stronger recirculation velocity, compared with the experimental measurements and with a stronger recirculation velocity. Figure 9 displays the comparisons of the mean wall-normal velocity profiles between coarse and fine grid LESs. The downwash recognized by the negative sign of the mean wall-normal velocity is captured well by both the LES and RANS computations. The ejected flow (as described by Devenport and Simpson [2]), characterized by the positive sign, is also well predicted at the first two stations. The RANS calculation result shows the opposite sign of the mean vertical velocity with the experimental measurement close to the bottom wall at the eighth station, whereas LES shows good agreement with the experimental data at that station.

Figure 10 displays the contours of turbulent kinetic energy (TKE) calculated with DES by Paik et al. [9], LES, and RANS, respectively. As shown in the experimental measurements, a peak is present near the core of the mean junction vortex. The maximum level of TKE is about 0.05 at $x / T=-0.2$. Moreover, contour lines have a characteristic $\mathrm{C}$ shape from $x / T=-0.2$ to -0.1 . The DES captures
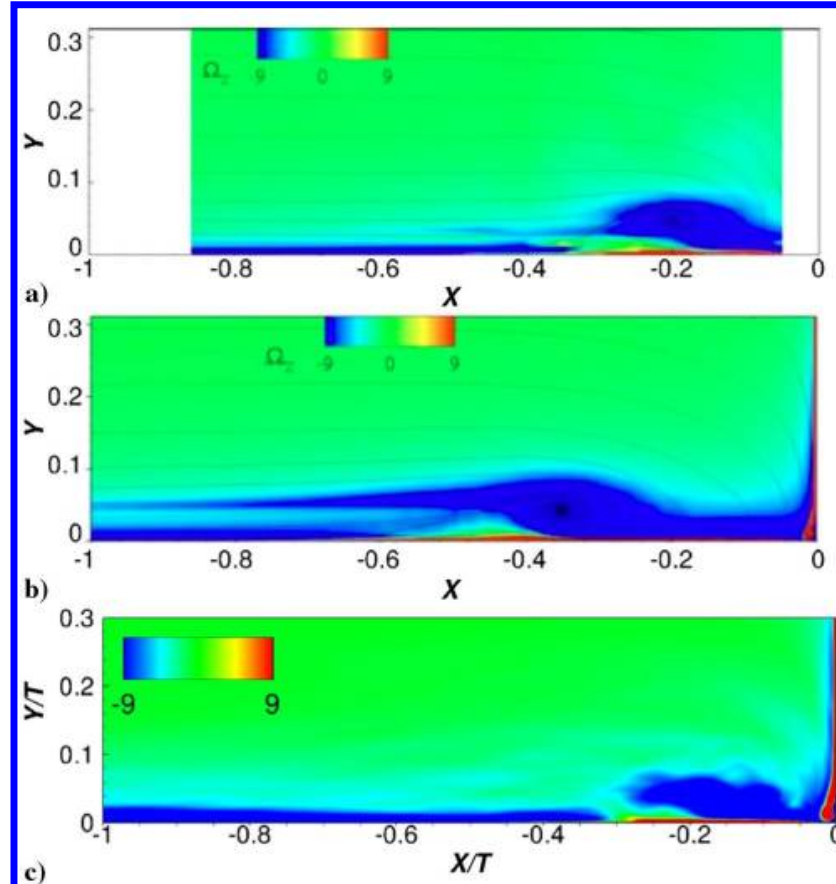

c
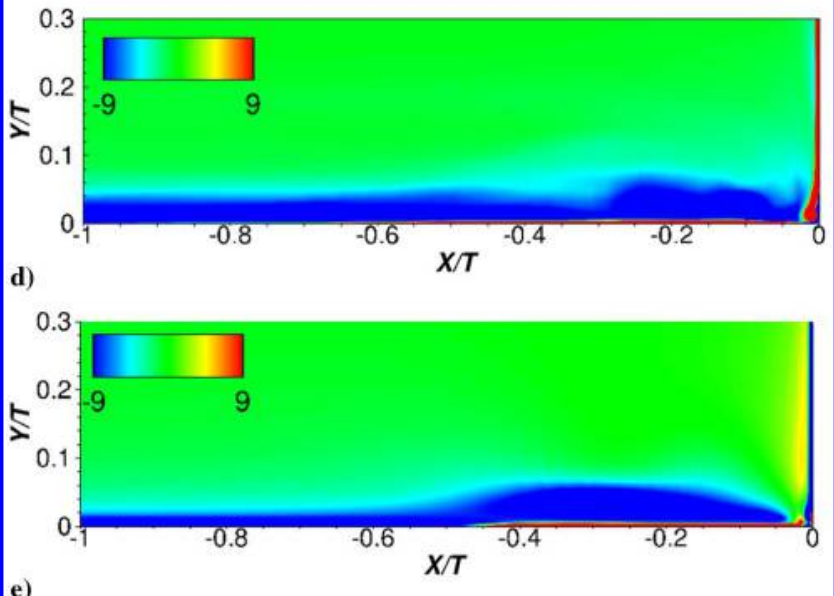

e)

Fig. 6 Time-averaged vorticity in spanwise direction $\Omega_{z}$ in symmetry plane upstream of airfoil. Vertical line at $x / T=0$ is airfoil leading edge: a) experimental measurements by Devenport and Simpson [2], b) DES by Paik et al. [9], c) LES with a fine grid, d) LES with a coarse grid, and e) RANS.

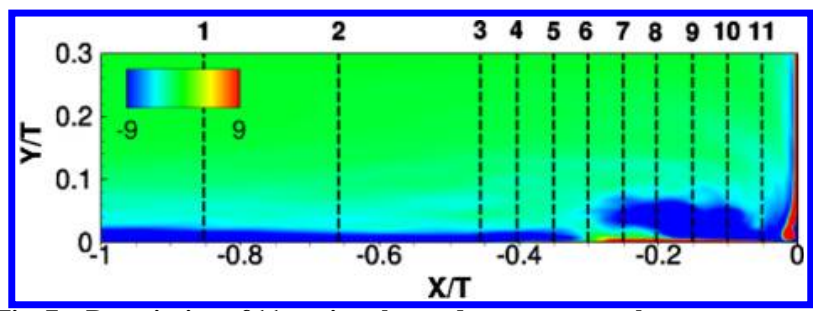

Fig. 7 Description of 11 stations located at symmetry plane upstream of airfoil. Experimental measurements [2] are available at the stations.

fairly well the characteristic structure of TKE and its strength, but the predicted structure is located further upstream compared with the experimental one, as shown in Fig. 10b. The LES leads to satisfactory predictions of the shape and strength of the TKE contours. It is notable that there exists a locally high TKE region in the core of the corner vortex, and its level is comparable to that observed in the core of the junction vortex. The TKE level calculated using RANS is about half that of the experiments. Moreover, the shape of the predicted TKE contour is qualitatively different. 


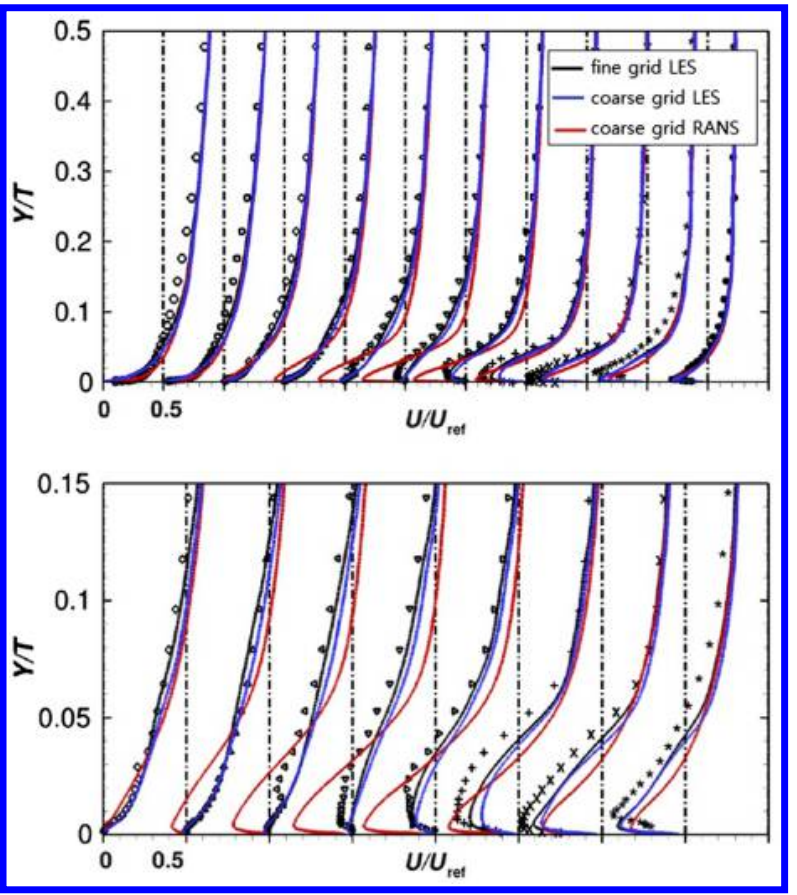

Fig. 8 Mean streamwise velocity profiles at 11 stations described in Fig. 7. Symbols, experimental measurements in [2]. Bottom figure presents a closer look at the near wall region.

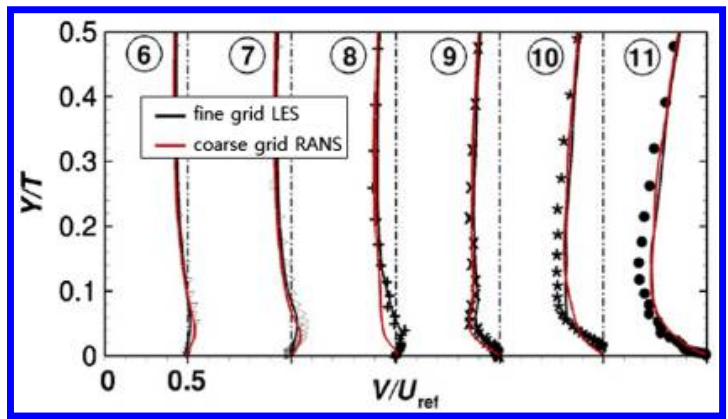

Fig. 9 Wall-normal mean velocity profiles at last six stations located in the plane of symmetry. Symbols, experimental measurements in [2].

Figure 11 shows the comparisons of rms values of the stremawise and vertical velocity fluctuations between coarse and fine grid LESs. As mentioned in Sec. III.C, interactions between incoming and reverse flows lead to low-frequency oscillations in the separation zone where the flow ejection occurs. The subsequent shear layer instability (see Paik et al. [9], figure 10), where the ejected flow is transported by the incoming flow momentum, leads to the characteristic variation of turbulence quantities shown in Fig. 11. The flow close to the wall is unstable, owing to the inflectional shear instability at the interface between reverse and incoming flow. Consequently, the peak location of the streamwise turbulence quantity is moved toward the wall, and its maximum value is increased at the seventh station. The profile of the streamwise turbulence quantity shows a second peak at the eighth station in the region dominated by the shear instability. Closer to the airfoil surface, the streamwise turbulence is damped, as can be shown in the 10th and 11th stations.

The rms of the vertical fluctuations gradually increase along the centerline $(y / T=0.05)$ of the mean vortex structure in the separation zone (from the sixth to the ninth station); afterward, these are reduced at the 10th and 11th stations, where wall-damping effects are larger than the turbulence production.

The LES is seen to capture fairly well the streamwise fluctuations at the eighth station. In general, the streamwise velocity fluctuations close to the wall are well predicted. However, the second peak of the streamwise turbulent fluctuation is not captured at the eighth station.

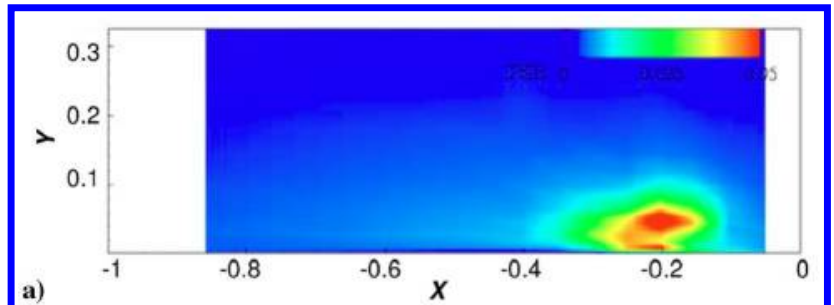

a)

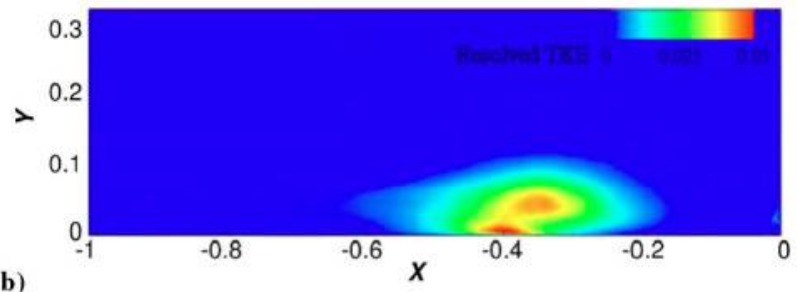

b)

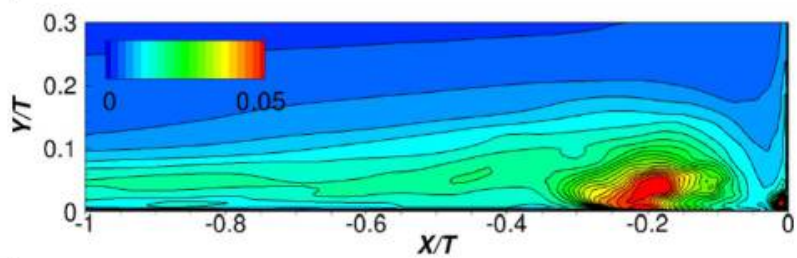

c)

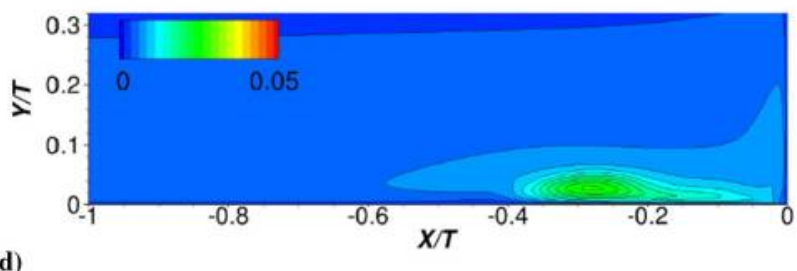

Fig. 10 Turbulent kinetic energy near junction: a), experimental measurements in [2], b) DES in [9], c) LES with a fine grid, and d) RANS.
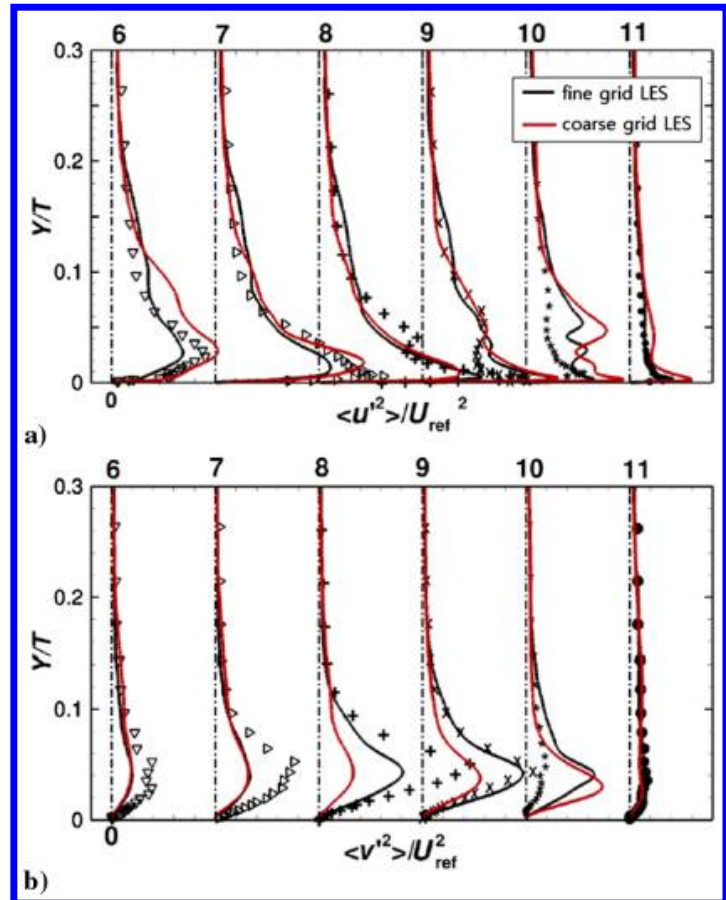

Fig. 11 Mean a) streamwise and b) wall-normal rms turbulence quantity at last six stations located in the plane of symmetry. Symbols, experimental measurements in [2].

Furthermore, the streamwise turbulence predicted by the LES is not sufficiently damped at the 10th station, in both the fine and coarse grid cases. The LES with a fine grid captures qualitatively the 
increase in the vertical turbulent fluctuations along the centerline, but highly overestimates it at the 10th station. Such discrepancies indicate that the Vreman model might be inaccurate for LES of wall junction flows because of the presence of strong pressure gradient effects, nonequilibrium effects, and complex wall damping. This motivates the sensitivity analysis carried out in the next section.

\section{E. Corner Flow Physics}

Figure 12 displays the secondary corner vortex structures visualized using streamlines and colored by the mean spanwise vorticity. The LES result calculated with a fine grid shows several structures that consist of a primary vortex and three secondary vortices. The rotating direction of the primary vortex is counterclockwise because the right branch of the downwash at $x / T=-0.025$ turns along the corner. Induced by the primary vortex, a counter-rotating secondary vortex is generated very close to the corner. Moreover, two secondary vortices are also generated near the primary vortex. Although the flow momentum of the downwash flow is mainly responsible for the generation of the primary vortex, the generation mechanism of the adjacent vortices is due to the interaction between boundary-layer flows turning upward from the corner and downward from the upper region on the wing nose. The rotational sense of the secondary vortex observed at $x / T=$ -0.002 and $y / T=-0.02$ is clockwise, whereas the sense of the vortex located at $x / T=-0.005$ and $y / T=-0.035$ is counterclockwise. Two stagnation points are observed at $x / T=-0.008$ and $y / T=0.028$, and $x / T=-0.002$ and $y / T=0.048$.

The LES result calculated with a coarse grid captures the primary vortex and the secondary corner vortex. However, the secondary vortex located at $x / T=-0.005$ and $y / T=-0.035$ is weaker than the fine grid case. Moreover, the secondary one observed at $x / T=$ -0.02 and $y / T=-0.02$ in the fine grid case is not captured in the coarse grid. The RANS calculation with the SST $k-\omega$ model reproduces the primary vortex and shows several discrepancies: the core of the primary vortex is located closer to the leading edge; its shape is more elliptic than those of the LES cases; and its strength is weaker than those of the LES cases. Moreover, the RANS calculation does not predict any secondary vortices. This is not surprising, given that linear eddy-viscosity models are limited in their ability to accurately predict secondary motions induced by the anisotropy of normal Reynolds stresses, as recently observed by Mani et al. [20].

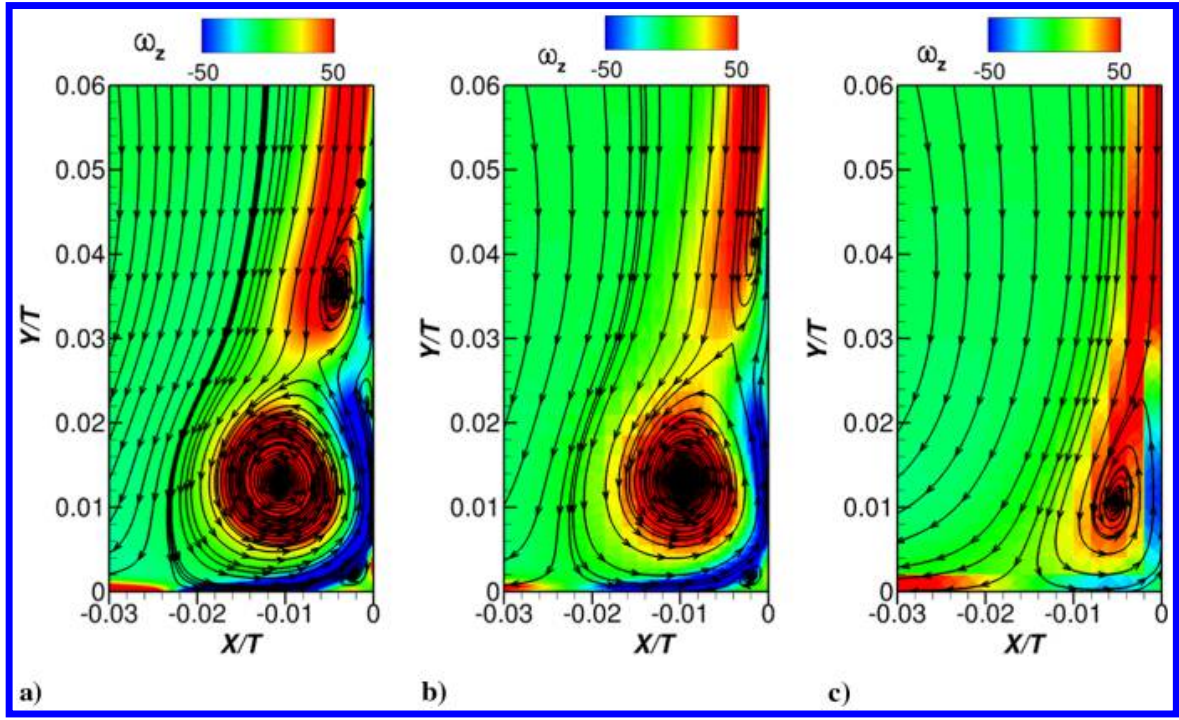

Fig. 12 Streamlines generated by mean streamwise and wall-normal velocity components. Color contours display the vorticity component out of plane: LES with a) fine and b) coarse grids; c) RANS with SST $k-\omega$.

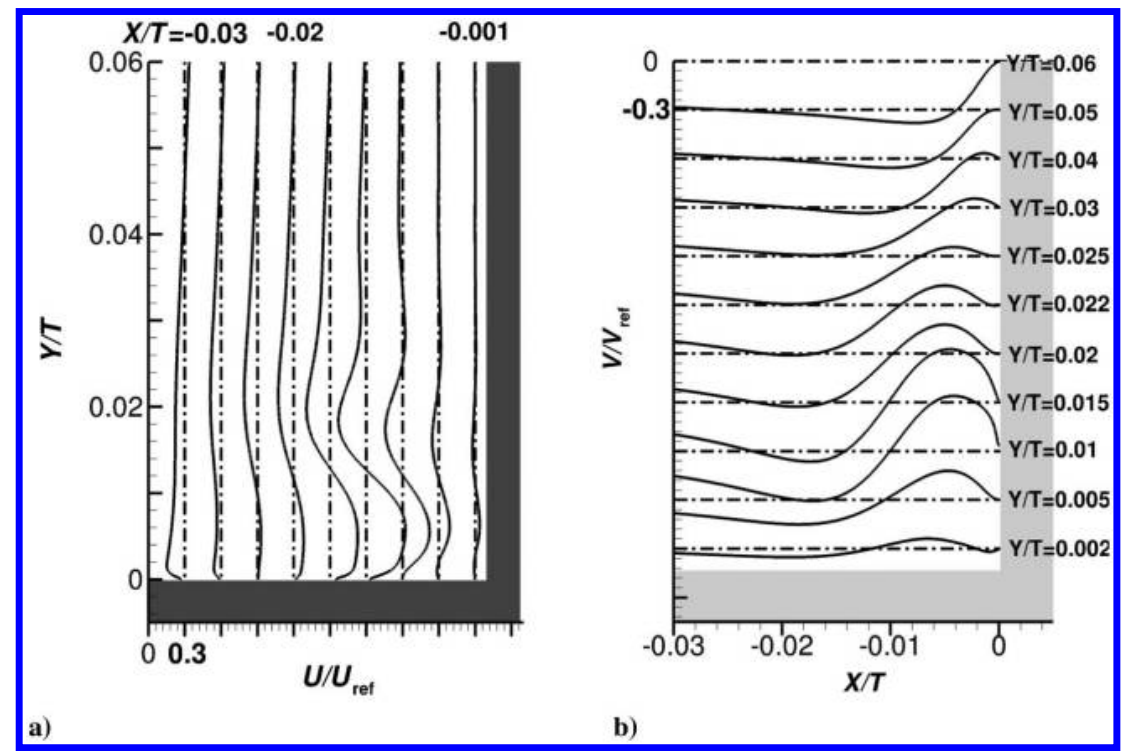

Fig. 13 Mean velocity profiles calculated by LES with a fine grid and extracted in the corner region: a) mean streamwise velocity at multiple streamwise stations; b) mean vertical velocity profiles at multiple vertical stations. 


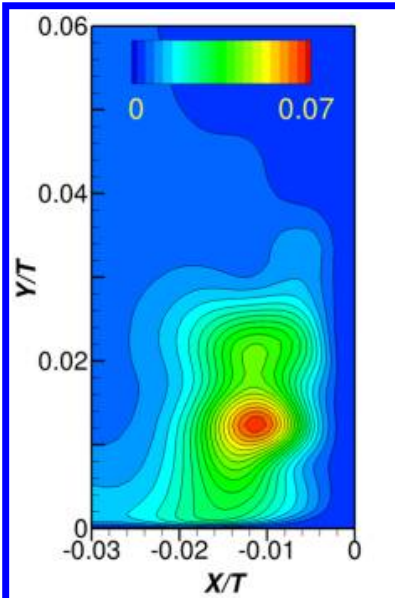

a)

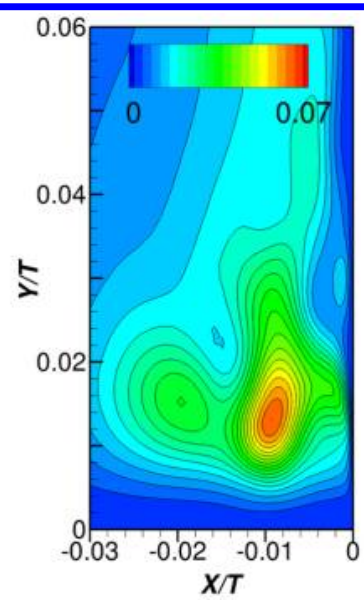

b)

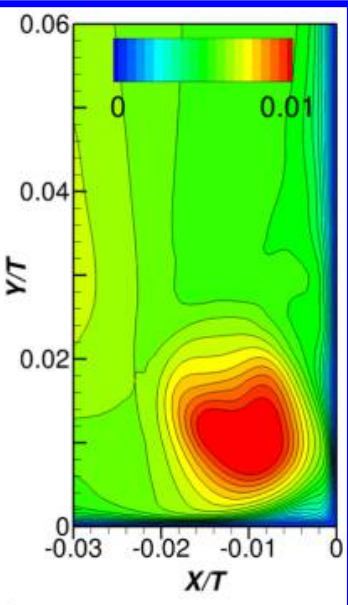

c)

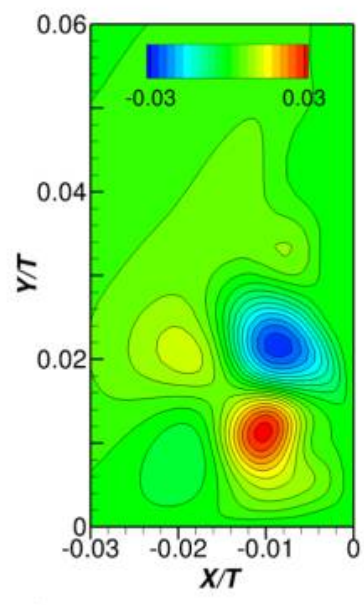

e)

Fig. 14 Contour of Reynolds stresses in the corner region: a) streamwise $\left(u^{\prime 2}\right)$, b) vertical $\left(v^{\prime 2}\right)$, and c) spanwise $\left(w^{\prime 2}\right)$ Reynolds normal stress; d) TKE $\left(1 / 2 \overline{u_{i}^{\prime} u_{i}^{\prime}}\right)$; and e) Reynolds shear stress $\left(\overline{u^{\prime} v^{\prime}}\right)$.

Figures $13 \mathrm{a}$ and $13 \mathrm{~b}$ display the mean streamwise and vertical velocity profiles in the corner region. The right branch of the downwash flow is recognized from the third to the eighth station close to the bottom wall, as shown in Fig. 13a. The boundary-layer flow turning along the corner is confirmed by the positive mean vertical velocity in the last five stations in Fig. 13b. The reverse flow turning upward is damped by interactions with the downwash flow. In such a turning process, the primary corner vortex is generated at $x / T=-0.01$ and $y / T=0.015$, where the mean streamwise and vertical velocity profiles are most inflectional. The generation mechanism of the secondary vortex located at $x / T=-0.005$ and $y / T=0.035$ is slightly different from that of the primary one.

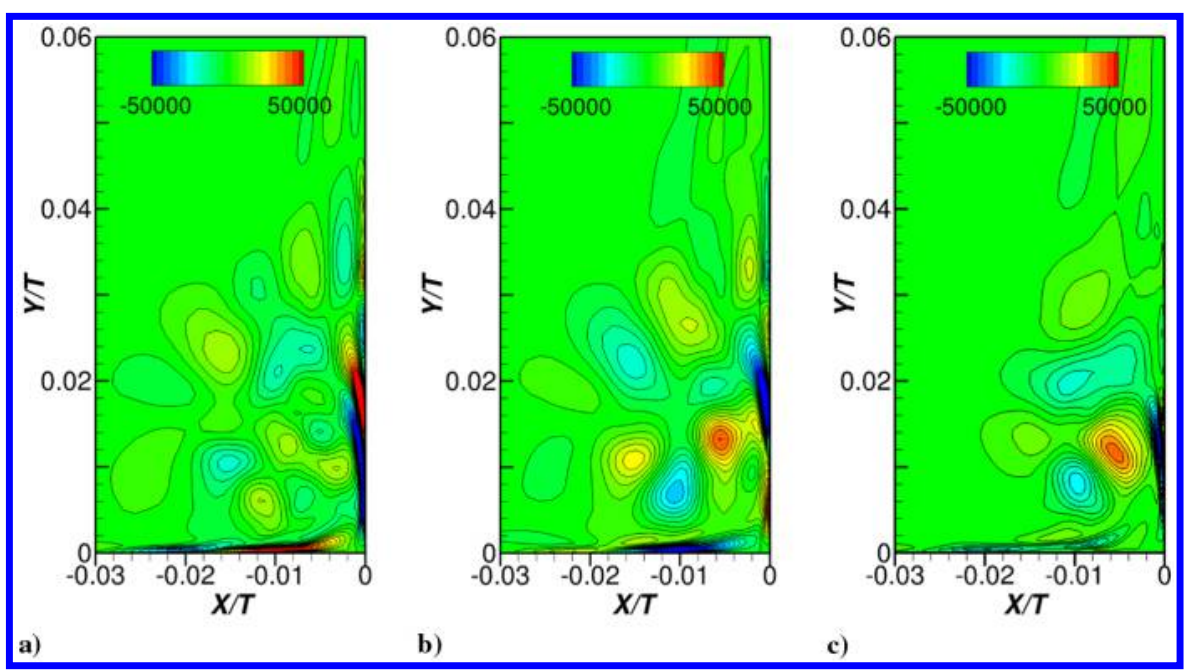

Fig. 15 Mean spanwise vorticity source terms calculated with fine grid LES data: a) normal Reynolds stress imbalance-induced source term $\left[\partial^{2}\left(v^{\prime 2}-u^{\prime 2}\right) / \partial x \partial y\right]$, b) spatial gradient of Reynolds shear stress-induced source term $\left[\left(\partial^{2} / \partial x^{2}-\partial^{2} / \partial y^{2}\right) \bar{u}^{\prime} v^{\prime}\right]$, c) sum of Figs. 15a and 15b. 
Although the primary vortex is generated by interactions between streamwise and vertical boundary-layer flows, the secondary one is formed by viscous interaction between the vertical boundary layer and the wall-mounted airfoil surface. This can be confirmed by the fact that the vertical mean velocity profile at the core location $(x / T=-0.005$ and $y / T=0.035)$ is much more inflectional than the streamwise mean velocity profile. Moreover, the other counterrotating secondary vortices are accompanied with the tiny separations, which are recognized at the last two streamwise stations and the seventh vertical station, respectively.

To investigate the generation mechanism of mean secondary flows very close to the corner region, the incompressible mean spanwise vorticity transport equation is considered as

$$
\begin{aligned}
& U \frac{\partial \Omega_{z}}{\partial x}+V \frac{\partial \Omega_{z}}{\partial y}=\frac{\partial^{2}}{\partial x \partial y}\left(\overline{v^{\prime 2}}-\overline{u^{\prime 2}}\right)+\left(\frac{\partial^{2}}{\partial y^{2}}-\frac{\partial^{2}}{\partial x^{2}}\right)\left(-\overline{u^{\prime} v^{\prime}}\right) \\
& \quad+\nu\left(\frac{\partial^{2} \Omega_{z}}{\partial x^{2}}+\frac{\partial^{2} \Omega_{z}}{\partial y^{2}}\right)
\end{aligned}
$$

where the mean spanwise velocity is assumed negligible. As seen in the right of Eq. (1), two mean vorticity source terms related to Reynolds stresses generate secondary flows, as discussed in Bradshaw [21]. Figure 14 displays the distribution of Reynolds stresses and turbulent kinetic energy, and these provide physical insights for analyzing the corner secondary flows. As seen in Figs. $14 \mathrm{a}$ and $14 \mathrm{~b}$, the normal Reynolds stresses $\left(\overline{u^{\prime} u^{\prime}}, \overline{v^{\prime} v^{\prime}}\right)$ show different wall-damping behaviors toward the wall-mounted wing surface and on the flat plate, respectively, very close to the corner region. Two different kinds of wall dampings are involved in the different wall behaviors of the two Reynolds normal stresses: viscous damping in wall-parallel flow and an inviscid one in wallnormal flow (or stagnation flow). In detail, the streamwise velocity is damped by the viscous friction on the bottom wall, whereas inviscid damping is dominant on the wing surface where a stagnation point locates. These different wall-damping mechanisms bring about imbalance of the Reynolds normal stresses $\left(\overline{u^{\prime} u^{\prime}}-\overline{v^{\prime} v^{\prime}}\right)$ near the corner region and further generate mean secondary flows. Figure 15a shows the anisotropic wall-damping-induced mean vorticity source term. As expected, the source term is strong close to the wing surface and bottom wall. In addition, the spatial gradient of Reynolds shear stress is also a source for mean secondary flows. Figure $15 \mathrm{~b}$ displays the second source term in the right of Eq. (1) and it is strongly negative near the wing surface and bottom wall close to the corner region. Figure $15 \mathrm{c}$ shows the sum of the two source terms, the structure of which can be separately interpreted into the near-wall structure and the core region of the primary corner vortex (Fig. 12a). Comparing Fig. 12a with Fig. 15c, the "hooklike" mean negative spanwise vorticity band (displayed in Fig. 12a), which characterizes the mean corner flow separation, shows a similar near-wall structure to that of the net source term. In the core region, the second source term contributes to the mean secondary flows more than the first one. As mentioned in Gatski and Rumsey [22], linear eddy-viscosity models cannot accurately capture the anisotropy of Reynolds normal stresses and further their induced secondary flows. Thus, the inaccurate prediction of the imbalance $\left(\overline{u^{\prime} u^{\prime}}-\overline{v^{\prime} v^{\prime}}\right)$ can be a reason for the low strength of the mean negative vorticity band (Fig. 12c) predicted by the SST $k$ - $\omega$ model. Besides, the accurate prediction of inviscid/viscous wall-damping interaction can be an interesting issue that should be considered in characterization of the performance of nonlinear RANS models.

\section{F. Transient Behavior of Vortex Structure}

Figure 16 illustrates the "bimodal dynamics" [2] of the junction vortex near the leading edge by five snapshots of the instantaneous spanwise vorticity field. In the first snapshot of the vorticity field at the plane of symmetry, an instantaneous junction vortex structure is observed at $x / T=-0.2$. This vortical structure moves upstream due to the reverse flow induced by the adverse pressure gradient. A stagnation point (so-called zero-flow mode in Devenport and Simpson [2]) is apparent at about $x / T=-0.2$, and a flow ejection occurs, as illustrated in the third snapshot of Fig. 16. The ejected flow is then convected downstream by the incoming stream away from the wall (so called backflow mode [2]). A Kelvin-Helmholtz instability breaks down the corresponding shear layer, as illustrated in the fourth and fifth time instance. The presence of the ejection and successive instability determine the mean flow structure and spatial variations of turbulence quantities discussed in Secs. III.C and III.D.

In addition to the strong horseshoe vortex and corresponding recirculation region upstream of the airfoil, an analysis of the nearwall region defined by the flat plate and the airfoil reveals the presence of a secondary vortex. This was not studied by Devenport and Simpson [2]. Figure 17 displays the dynamics of this secondary corner vortex with six time instances of the spanwise vorticity field. In Fig. 17a, the periodic dynamics of the corner vortex is illustrated. Initially, the core shape is fairly circular, then the vertical downward motion along the airfoil leading edge stretches it in an elliptical form. Finally, the vortex interacts with the bottom wall and recovers the circular shape.
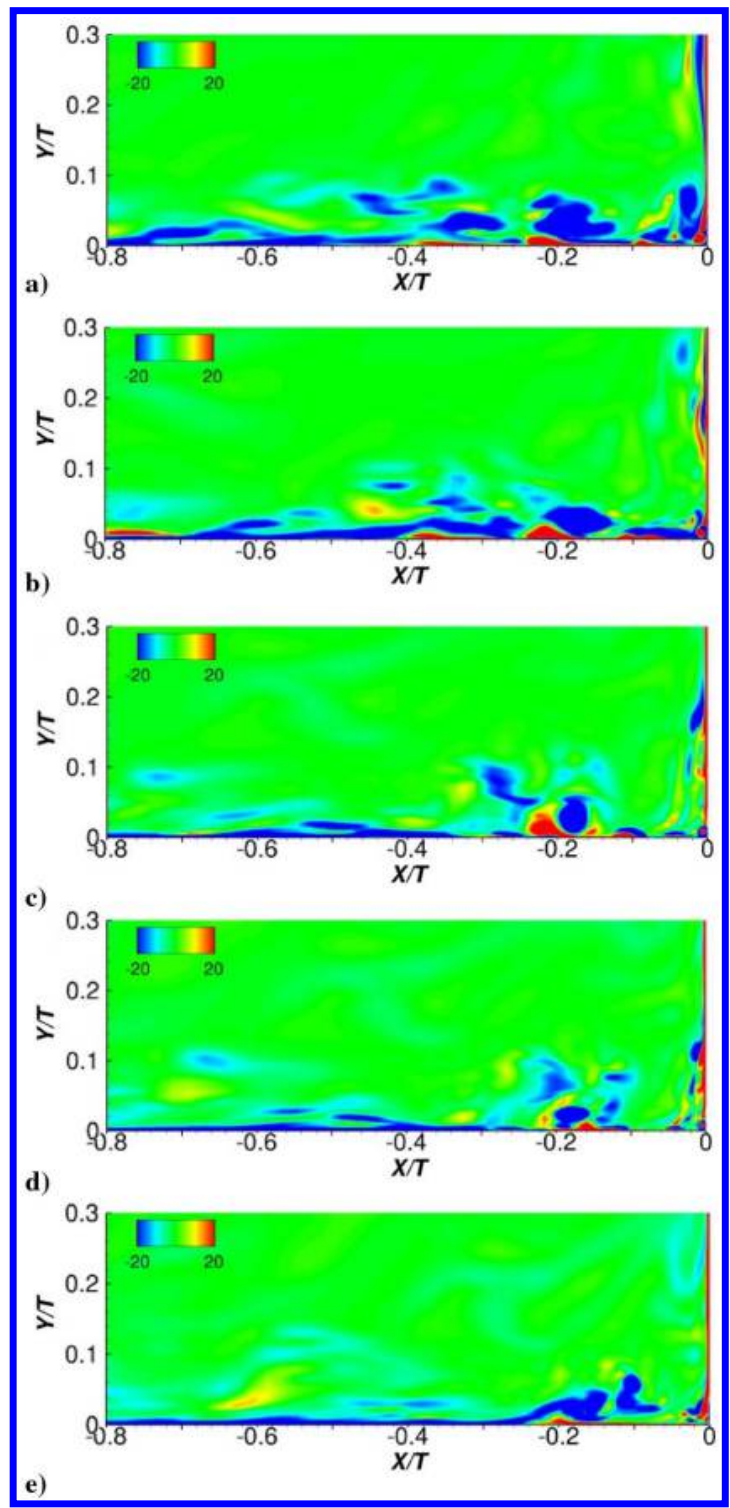

Fig. 16 Snapshots of instantaneous spanwise vorticity in the plane of symmetry near leading edge at a) $t_{0}$, b) $t_{0}+0.92$, c) $t_{0}+1.84$, d) $t_{0}+2.30$, and e) $t_{0}+3.22$. Time $t$ is normalized by reference time scale $T / U_{\text {ref }}$. 


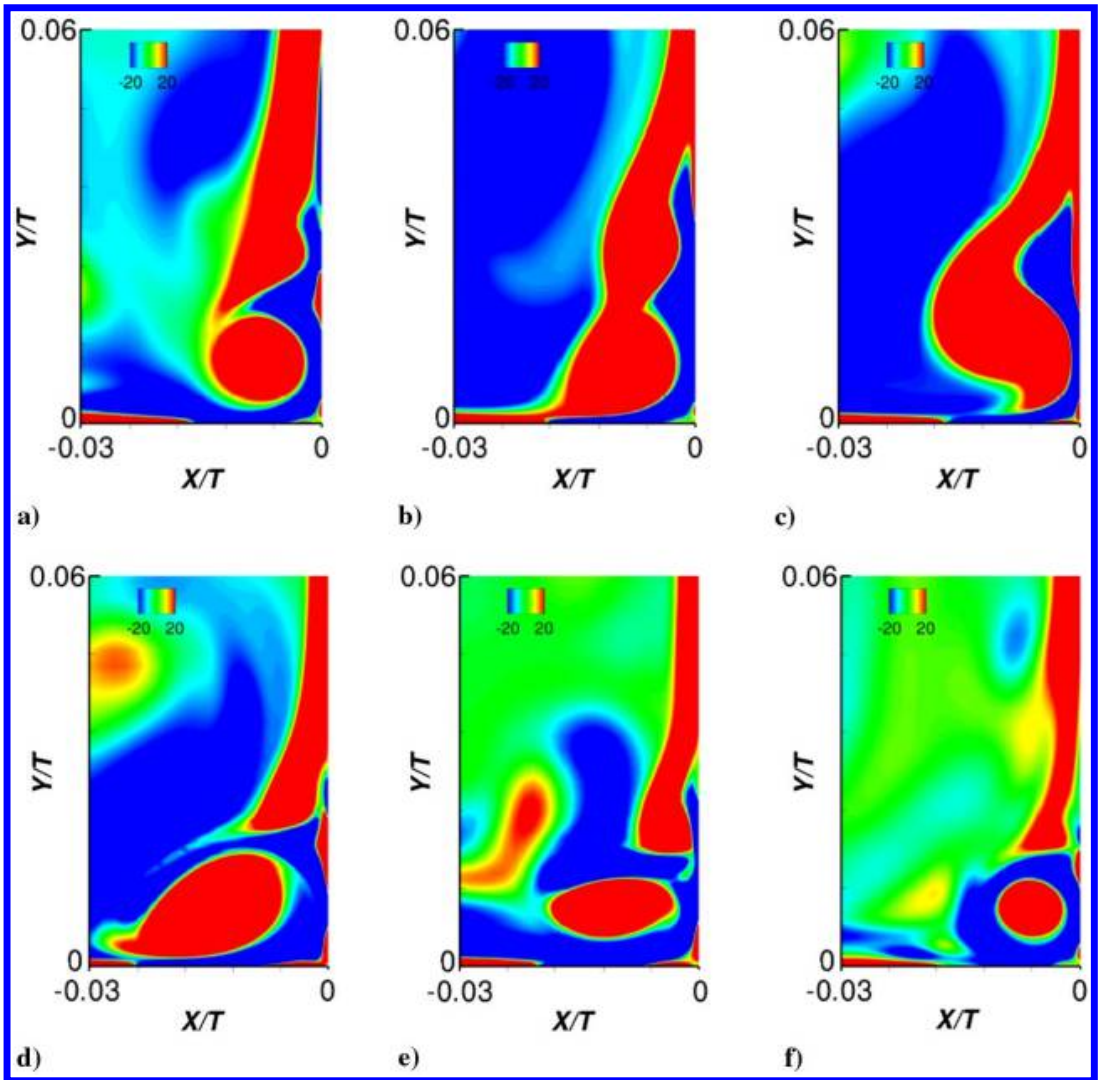

Fig. 17 Snapshots of instantaneous spanwise vorticity in the plane of symmetry at the corner region at a) $t_{0}$, b) $t_{0}+0.55$, c) $t_{0}+0.74$, d) $t_{0}+0.92$, e) $t_{0}+1.38$, and f) $t_{0}+2.12$.

\section{Sensitivity Analysis}

\section{A. Sensitivity Analysis of Inlet Condition}

Three different inlet source profiles are tested with LES with a fine grid to carry out sensitivity analysis on the generated turbulent inlet profiles: one-dimensional (1-D) profiles experimentally measured by Devenport and Simpson [2] at $x / T=-18.24(x / c=-4.287), 1-\mathrm{D}$ fully developed profiles calculated with RANS at $x / T=-4.254$ $(x / c=-1)$, and 1-D fully developed profiles calculated with RANS at $R e_{\theta}=5940$. The 1-D profiles experimentally measured by Devenport and Simpson [2] at $x / T=-18.24(x / c=-4.287)$ include only three components $\left(U,\left\langle u^{\prime} u^{\prime}\right\rangle,\left\langle w^{\prime} w^{\prime}\right\rangle\right)$, and the inlet location $x / T=4.254(x / c=-1)$ of the computational domain is located downstream with respect to the measurement location. The 1D fully developed profiles calculated with the 1-D RANS solver at $x / T=-4.254(x / c=-1)$ are used to prescribe the turbulent inlet condition. The $1-\mathrm{D}$ profiles include five components $\left(U,\left\langle u^{\prime} u^{\prime}\right\rangle\right.$, $\left.\left\langle v^{\prime} v^{\prime}\right\rangle,\left\langle w^{\prime} w^{\prime}\right\rangle,\left\langle u^{\prime} v^{\prime}\right\rangle\right)$. Last, the 1-D fully developed profiles calculated with 1-D RANS solver at $R e_{\theta}=5811$ are imposed for the synthesized inlet to match the experimental conditions. The $R e_{\theta}=$ 5811 is approximated from the $R e_{\theta}=5940$ measured by Ölçmen and Simpson [4] at $x / c=-0.75$ (inlet and measurement locations are described in Fig. 2), using the relation between development length Reynolds number $R e_{x}$ and momentum thickness $\theta$ on the flat plate as

$$
\frac{\theta}{x}=\frac{0.0367}{R e_{x}^{0.2}}
$$

Figure 18 displays time-averaged mean velocity profiles calculated with the three different inlet conditions. The 11 profile stations are located in the plane of symmetry. The mean streamwise velocity profiles calculated with the first inlet condition show early flow separation at the second station and mismatch of the boundarylayer thickness $\delta_{99}$ with the experimental measurement at the first five stations. In the second case, the early separation problem is fixed by the computed 1-D source profiles, but the predicted boundary-layer thickness is roughly two times larger than the measured one at the first five stations. In the last case, the early separation and mismatch of the boundary-layer thickness are remedied. Moreover, the freestream velocities at the 11 stations are well reproduced.

Figure 19 shows comparisons of the streamwise turbulence quantity corresponding with the three inlet cases. In the first and second cases, the computed turbulence quantity significantly deviates from the experimental measurement in the separation zone (sixth-ninth stations). However, the third case shows reasonable agreements with the measurements in the last seven stations, although the turbulence quantity is slightly overpredicted in the first three stations.

\section{B. Investigation of SGS Modeling Effect}

LESs of the wing-body junction case are performed with the Vreman model, the VSS-model [23], and the dynamic Smagorinsky model (DSM) [24] to investigate SGS modeling effects on the turbulence statistics in the symmetry plane. The coarse grid is used for the calculations, and simulation conditions for the LES are exactly same with those of the case presented in Sec. II. Clipping is used to stabilize a spatially steep change of the model constant calculated with the Germano identity and the least-square-based modification [25] in the DSM. The turbulence statistics are accumulated for 1.5 flowthrough times.

Figures 20a and 20b display the mean streamwise and vertical velocity profiles calculated with the three SGS models. The mean velocities are compared in the last six stations where the mean flow separation is observed in the fine grid LES results. At station 6, the DSM slightly overpredicts the amount of reverse flow (about two times larger than the other models). Qualitatively, the saddle points of the mean streamwise velocities predicted by the three models are located closer to the bottom wall than those of the experimental data at station 7. The amount of reversed flow is small compared with the 


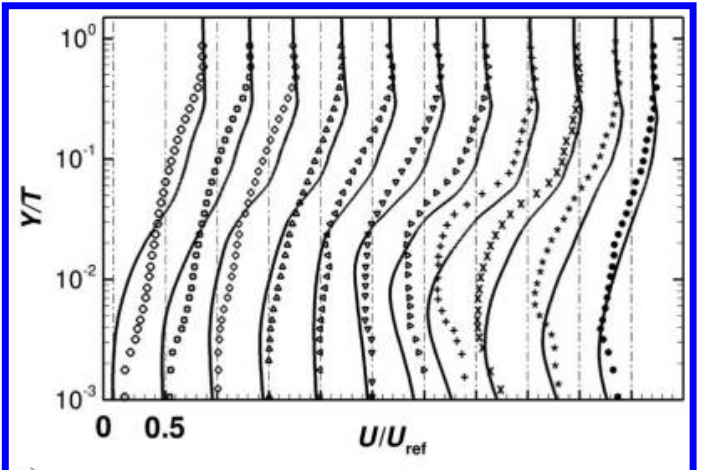

a)

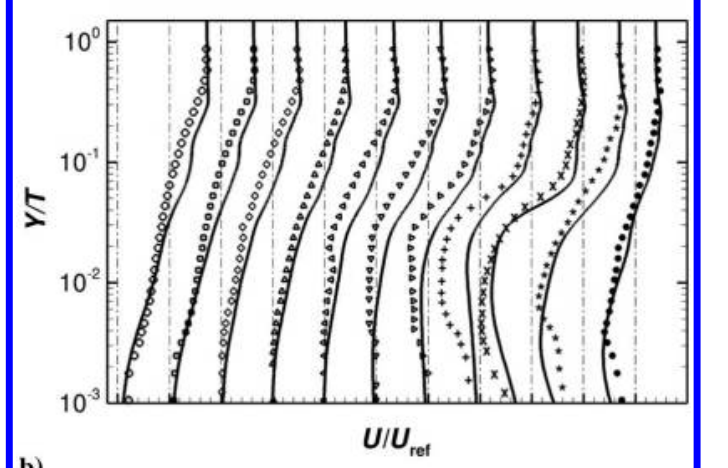

b)

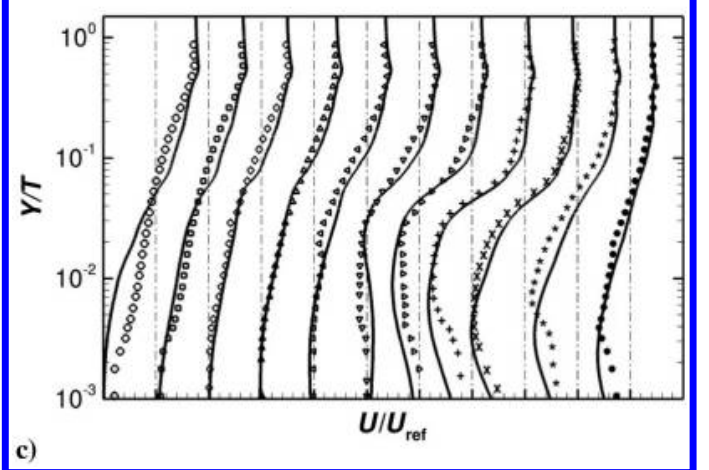

Fig. 18 Mean streamwise velocity profiles at multiple stations described in Fig. 7 with respect to three different 1-D inlet source profiles: a) LES results with synthesized inlet conditions with 1-D profiles measured by Devenport and Simpson [2] at $x / T=-18.24(x / c=-4.287)$; b) 1-D fully developed profiles calculated by 1-D RANS solver at $x / T=-4.254$ $(x / c=-1)$; and c) 1-D fully developed profiles calculated with 1-D RANS solver at $\operatorname{Re}_{\theta}=5811$.

experimental data in stations $8-10$. Figure $20 \mathrm{~b}$ shows comparisons of the mean vertical velocity profiles with respect to the three SGS models. The downwash generated by the wall-blocking effect is qualitatively well predicted by the three models in the six stations, but the flow ejection recognized by the positive sign of the mean vertical velocity is not accurately captured by the models at the first three stations.

Figure 21a shows the streamwise rms turbulence quantities computed with the three SGS models. Unlike the cases of the mean velocity profiles, the qualitative differences induced by SGS modeling effects are pronounced in the comparison of rms values. As mentioned in Sec. III.D, the Vreman model does not predict the second peak in the upper region at stations 8 and 9 . Moreover, the Vreman model does not capture the turbulence damping in station 10 in the fine grid case. Qualitatively, the characteristic double-peak variation (mentioned in Sec. III.D) is well predicted by the Vreman model and the VSS model in station 8, whereas these peaks are not shown in the DSM results. However, the DSM recovers the characteristic profiles at station 9. In station 10, the VSS and the DSM

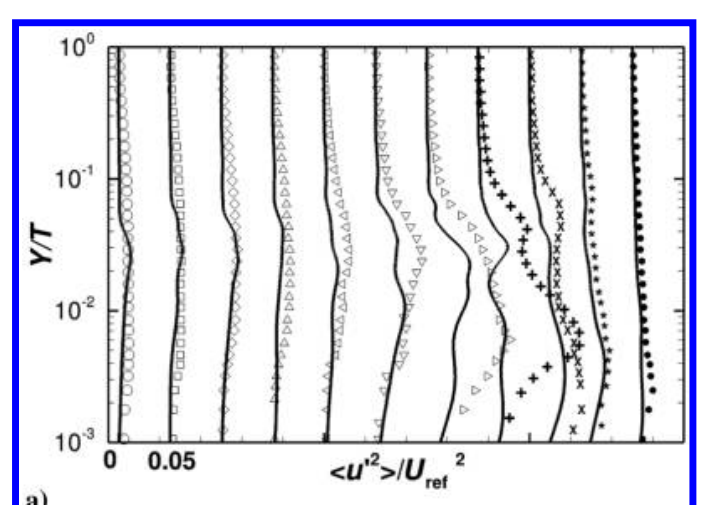

a)

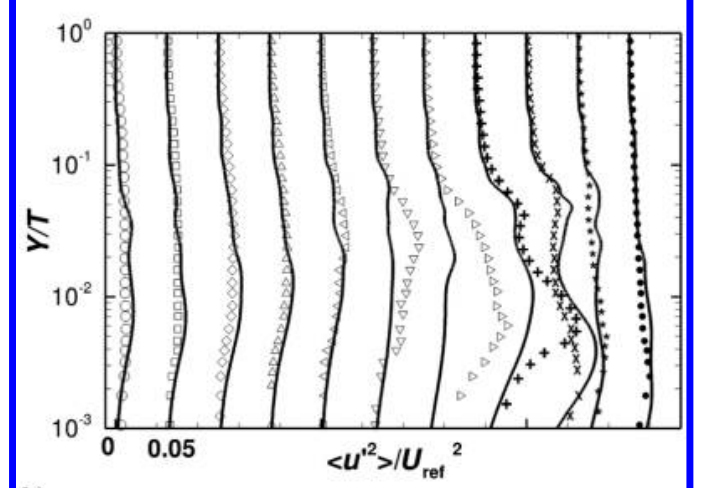

b)

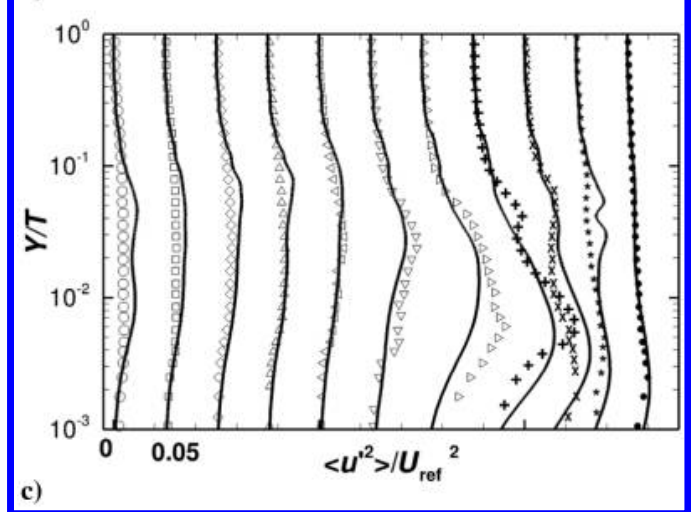

Fig. 19 Same as Fig. 18, but streamwise turbulence fluctuations.

show the smooth streamwise turbulence damping in the vertical direction. The characteristic variation shown in station 9 is not completely damped by the Vreman model at station 10, as shown in Fig. 21a.

Figure $21 \mathrm{~b}$ displays the comparison of the vertical velocity fluctuations. As mentioned in Sec. III.D, the gradual increase and damping of the vertical turbulence are the main characteristics shown in the experimental data at the symmetry plane. Although the Vreman model on the coarse grid does not show turbulence damping in station 10 (which was observed on the fine grid in Sec. III.D), the other SGS models qualitatively recover the damping of the vertical turbulence quantity. Although the three SGS model results do not show qualitatively distinguished differences from the experimental data between stations 6-8, the DSM and the VSS are both clearly superior to the Vreman model in the prediction of the vertical turbulence damping at station 10. In other words, the vertical turbulence predicted by the Vreman model continuously increases from stations 6-10, whereas those of the other models start to be damped from station 8 . At station 11, all models are able to recover the turbulence damping behavior. The different predictions of the vertical rms turbulence damping could be caused by the different wall behaviors of the SGS models (i.e., the Vreman model shows the linear wall behavior, unlike the cubic wall behavior of the other models $[\underline{23}, \underline{26}])$. 


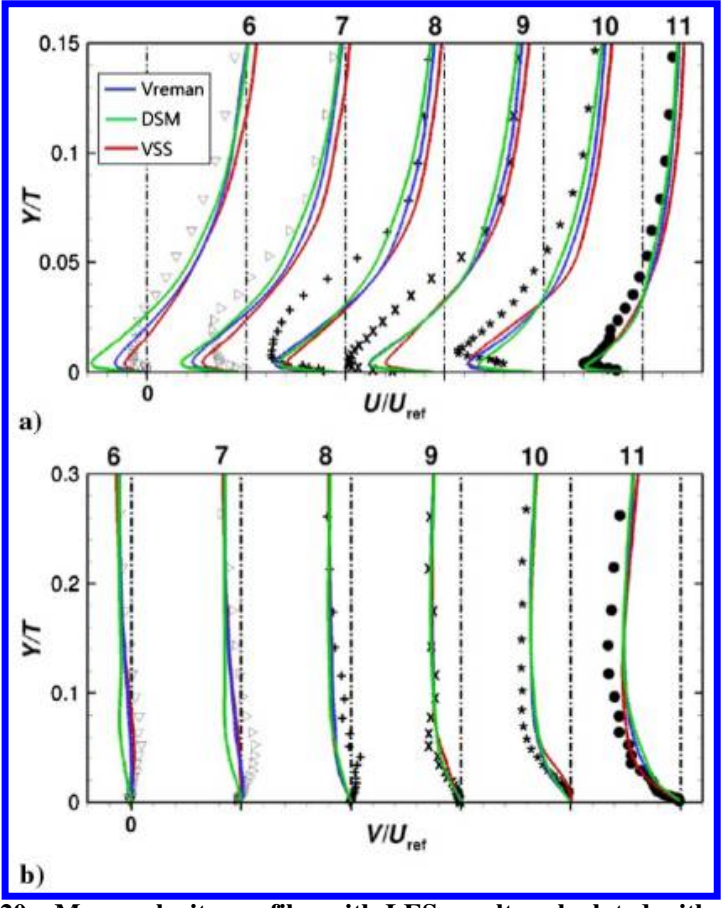

Fig. 20 Mean velocity profiles with LES results calculated with three SGS models: a) mean streamwise velocity and b) mean vertical velocity. Black symbols, experimental data from [2].
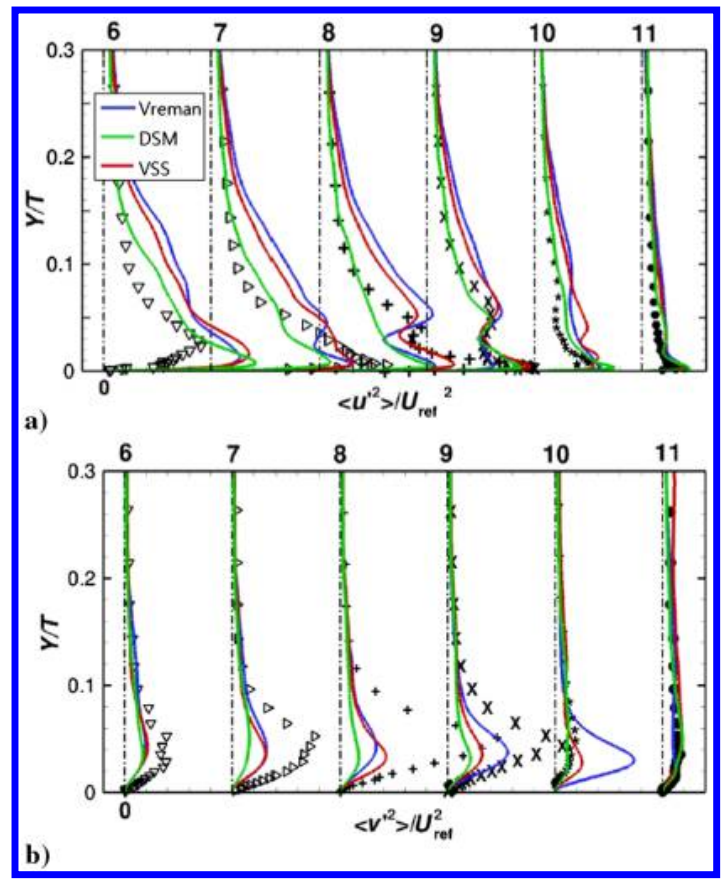

Fig. 21 Root mean square turbulence quantities with LES results calculated with three SGS models: a) streamwise and b) vertical rms turbulence quantities. Symbols and lines same as Fig. 20.

\section{Conclusions}

Detailed LESs of the wing-body junction case, experimentally investigated by Devenport and Simpson [2], have been presented. The LES results are compared with experimental measurements, DES [9], and RANS with the SST $k-\omega$ model, investigating mean flow structure and turbulence statistics near the junction in the plane of symmetry. First, the mean flow structure, characterized by the mean spanwise vorticity, is evaluated near the junction. Whereas Although the LES captures the elliptic mean vortex structure and its location, the DES incorrectly predicts the core location further upstream. The mean flow structure predicted by the RANS calculation shows a $30 \%$ wider separation zone than the experimentally measured one. Moreover, turbulence statistics are compared at 11 stations located in the symmetry plane to quantitatively validate the LES results. The LES captures fairly well the separation location and reverse flows generated by wall blocking effects. However, the LES with the Vreman model does not completely reflect the flow-structure-induced double peak profiles in the streamwise turbulence quantity or streamwise and vertical turbulence damping close to the junction.

Using the LES data, corner flows not experimentally studied by Devenport and Simpson [2] are investigated. The temporal behavior of the corner vortex is analyzed with time sequences of the spanwise vorticity. Moreover, the mean corner vortex pattern, which consists of one primary vortex and three secondary vortices, is presented, and its generation mechanism is illustrated. Significant anisotropy of the Reynolds normal stresses and spatially anisotropic turbulence damping near the airfoil surface and the bottom wall are noted.

Sensitivity analysis for the body junction flow is carried out with respect to the inlet condition. First, three different inlet conditions are tested. The one corresponding to the base profile at $R e_{\theta}=5811$ shows the most accurate prediction of the mean streamwise velocity near the junction. The first inlet case results in early flow separation near the junction and thinner boundary-layer thickness in the outer layer. The second case resolves the early separation problem shown in the first case, but the predicted boundary-layer thickness is half as thick the experimental one. The third case resolves the two inaccurate predictions (thin boundary-layer thickness and early flow separation).

SGS modeling effects on the turbulence statistics are studied in the LES computed with the Vreman model, the VSS, and the DSM. Qualitatively, the three SGS models show different performance, especially in the flow structure-induced characteristic variation of the rms turbulence quantities and turbulence damping near the wing nose. It is notable that the Vreman model does not recover appropriate turbulence damping at a station very close to the wing nose, whereas the other models do.

\section{Acknowledgment}

This work is funded under NASA Cooperative Agreement NNX11AI41A with Steve Woodruff as technical monitor.

\section{References}

[1] Simpson, R. L., "Junction Flows," Annual Review of Fluid Mechanics, Vol. 54, No. 1, 2001, pp. 415-443. doi:10.1146/annurev.fluid.33.1.415

[2] Devenport, W. J., and Simpson, R. L., "Time-Dependent and TimeAveraged Turbulence Structure near the Nose of a Wing-Body Junction," Journal of Fluid Mechanics, Vol. 210, No. 1, 1990, pp. $23-55$ doi: $10.1017 / \mathrm{S} 0022112090001215$

-[3] Fleming, J. L., Simpson, R. L., Cowling, J. E., and Devenport, W. J., “An Experimental Study of a Turbulent Wing-Body Junction and Wake Flow," Experiments in Fluids, Vol. 14, No. 5, 1993, pp. 366-378. doi:10.1007/BF00189496

- [4] Ölçmen, S. M., and Simpson, R. L., "An Experimental Study of a ThreeDimensional Pressure-Driven Turbulent Boundary Layer," Journal of Fluid Mechanics, Vol. 290, No. 1, 1995, pp. 225-262. doi:10.1017/S0022112095002497

[5] Apsley, J. L., and Leschziner, W. J., "Investigation of Advanced Turbulence Models for the Flow in a Generic Wing-Body Junction," Flow, Turbulence and Combustion, Vol. 67, No. 1, 2001, pp. 25-55. doi:10.1023/A:1013598401276

[6] Parneix, S., Durbin, P. A., and Behnia, M., "Computation of 3-D Turbulent Boundary Layers Using V2F model," Flow, Turbulence and Combustion, Vol. 60, No. 1, 1998, pp. 19-46. doi:10.1023/A:1009986925097

[7] Lien, F. S., Kalitzin, G., and Durbin, P. A., "RANS Modeling for Compressible and Transitional Flows," Center for Turbulence Research: Proceedings of the Summer Program, Center for Turbulence Research, Stanford, 1998, pp. 267-286.

[8] Iaccarino, G., "Predictions of a Turbulent Separated Flow Using Commercial CFD Codes," Journal of Fluid Engineering, Vol. 123, 
No. 4, 2001, pp. 819-828 doi: $10.1115 / 1.1400749$

[9] Paik, J., Escauriaza, C., and Sotiropoulos, F., "On the Bimodal Dynamics of the Turbulent Horseshoe Vortex System in a Wing-Body Junction," Physics of Fluids, Vol. 19, No. 4, 2007, Paper 045107. doi:10.1063/1.2716813

[10] Gand, F., Deck, S., Brunet, V., and Sagaut, P., "Flow Dynamics Past a Simplified Wing Body Junction," Physics of Fluids, Vol. 22, No. 11, 2010, Paper 115111 doi:10.1063/1.3500697

[11] Pierce, F. J., and Tree, I. K., "The Mean Flow Structure on the Symmetry Plane of a Turbulent Junction Vortex," Journal of Fluids Engineering, Vol. 112, No. 1, 1990, pp. 16-22. doi:10.1115/1.2909361

[12] Pierce, F. J., and Shin, J., "The Development of a Turbulent Junction Vortex System," Journal of Fluids Engineering, Vol. 114, No. 4, 1992, pp. $559-565$ doi:10.1115/1.2910068

[13] Larrson, J., Vicquelin, R., and Bermejo-Moreno, I., "Large Eddy Simulations of the HyShot II Scramjet," Center for Turbulence Research Annual Research Briefs, Center for Turbulence Research, Stanford, 2011, pp. 63-74.

[14] Vreman, A. W., "An Eddy-Viscosity Subgrid-Scale Model for Turbulent Shear Flow: Algebraic Theory and Applications," Physics of Fluids, Vol. 16, No. 10, 2004, pp. 3670-3681. doi:10.1063/1.1785131

[15] Xie, Z. T., and Castro, I. P., "Efficient Generation of Inflow Conditions for Large Eddy Simulation," Flow, Turbulence and Combustion, Vol. 81, No. 3, 2008, pp. 449-470. doi:10.1007/s10494-008-9151-5

[16] Touber, E., and Sandham, N. D., "Large-Eddy Simulation of LowFrequency Unsteadiness in a Turbulent Shock-Induced Separation Bubble," Theoretical and Computational Fluid Dynamics, Vol. 23, No. 2, 2009, pp. 79-107. doi:10.1007/s00162-009-0103-Z

[17] Wilcox, D. C., Turbulence Modeling for CFD, 3rd ed., DCW Industries, La Cañada Flintridge, CA, 2006.

[18] Menter, F. R., Kuntz, M., and Langtry, R., "Ten Years of Industrial Experience with the SST Turbulence Model," Turbulence, Heat and
Mass Transfer 4, edited by Hanjalik, K., Nagario, Y., and Tummers, M., Begell House, Redding, CT, 2003, pp. 625-632.

[19] Pecnik, R., Terrapon, V., Ham, F., Iaccarino, G., and Pitsch, H., "Reynolds-Averaged Navier-Stokes Simulations of the HyShot II Scramjet," AIAA Journal, Vol. 50, No. 8, 2012, pp. 1717-1732. doi:10.2514/1.J051473

[20] Mani, M., Bobcock, M., Winkler, C. M., and Spalart, P. R., "Predictions of a Supersonic Turbulent Flow in a Square Duct," 51st AIAA Aerospace Sciences Meeting Including the New Horizons Forum and Aerospace Exposition, AIAA Paper 2013-0860, 2013.

[21] Bradshaw, P., "Turbulent Secondary Flows," Annual Review of Fluid Mechanics, Vol. 19, No. 1, 1987, pp. 53-74. doi:10.1146/annurev.fl.19.010187.000413

[22] Gatski, T. B., and Rumsey, C. L., "Linear and Nonlinear Eddy Viscosity Models," Closure Strategies for Turbulent and Transitional Flows, edited by Launder, B., and Sandham, N., Cambridge Univ. Press, Cambridge, MA, 2001, pp. 9-46.

[23] Ryu, S., and Iaccarino, G., "A Subgrid-Scale Eddy-Viscosity Model Based on the Volumetric Strain-Stretching," Physics of Fluids, Vol. 26, No. 6, 2014, Paper 065107. doi: $10.1063 / 1.4882880$

[24] Germano, M., Piomelli, U., Moin, P., and Cabot, W. H., "A Dynamic Subgrid-Scale Eddy Viscosity Model," Physics of Fluids A: Fluid Dynamics, Vol. 3, No. 7, 1991, pp. 1760-1765. doi: $10.1063 / 1.857955$

[25] Lilly, D. K., "A Proposed Modification of the Germano Subgrid-Scale Closure Method," Physics of Fluids A: Fluid Dynamics, Vol. 4, No. 3 , 1991, pp. 633-635. doi: $10.1063 / 1.858280$

[26] Nicoud, F., Toda, H. B., Cabrit, O., Bose, S., and Lee, J., "Using Singular Values to Build a Subgrid-Scale Model for Large Eddy Simulations," Physics of Fluids, Vol. 23, No. 8, 2011, Paper 085106. doi:10.1063/1.3623274

M. Visbal Associate Editor 
This article has been cited by:

1. Wolfgang Schanderl, Ulrich Jenssen, Claudia Strobl, Michael Manhart. 2017. The structure and budget of turbulent kinetic energy in front of a wall-mounted cylinder. Journal of Fluid Mechanics 827, 285-321. [Crossref] 